\title{
Dissipation and Ellipticity of the Chandler Wobble
}

\author{
Yaozhong Zhu and Buxi Gao \\ Institute of Geodesy and Geophysics, Chinese Academy of Sciences, 54 \\ Xudong Road, Wuhan, Hubei, 430077, China
}

\begin{abstract}
The Chandler wobble, one of the main feature of the Earth's polar motion, is related to the properties of the mantle and liquid core as well as the mobility of the oceans. The equilibrium pole tide and mantle anelasticity both lengthen the Chandler period, moreover, the former imposes a slight ellipticity on the pole path, and the latter is responsible for the wobble energy dissipation. On the basis of the perturbation principles, we derive the theoretical $Q_{w}$ of the Chandler wobble, assuming that the wobble energy is totally dissipated within the mantle. The theoretical ellipticity and orientation of the semimajor axis of the Chandler wobble path for an anelastic Earth are given. Compared with the results for the elastic Earth, the effect of mantle anelasticity does not change the wobble ellipticity significantly, but slightly changes the orientation of the semimajor axis in the opposite direction. This paper has also proved that the effect of the Earth's 3-axis feature on the wobble ellipticity is only about $19 \%$ of that of the equilibrium pole tide. Analysis of the polar motion data obtained by using modern geodetic techniques shows that the observed ellipticity and orientation of the semimajor axis agree with the theoretical results. We can deduce that the pole tide in the globe should be close to equilibrium.
\end{abstract}

\section{Introduction}

The main feature of changes in the position of the rotation axis relative to the body of the Earth is the Chandler wobble. The period $T_{w}$ and quality factor $Q_{w}$ of the Chandler wobble are two of the fundamental parameters which describe the free polar motion, especially $Q_{w}$. It not only represents the dissipative ratio of the wobble energy, but also provides an important basis for studying the physical condition of the Earth's interior and establishing a united formula of $Q$ values on different time scales. The combined effect of the elasticity and anelasticity of the mantle, and the fluidity of the core and oceans leads to the observed Chandler wobble period of 435 sidereal days. Mantle anelasticity may play a major role in dissipating the wobble energy. The pole tide is the oceanic response to the change in centrifugal force accompanying the wobble. The equilibrium pole tide forces the pole path to be slightly elliptical.

The Chandler wobble period is well-known from astrometric observations within an accuracy of 2 to 3 days. The observed $Q_{w}$ is probably between 35 and 400 , with the preferred values of about 50-100 (Jeffreys 1968, Wilson \& 
Haubrich 1976, Ooe 1978, Kuehne et al. 1996, Furuya \& Chao 1996). Smith \& Dahlen (1981) calculate the theoretical Chandler period by modelling the Earth as a fluid core, an elastic mantle and equilibrium pole tide, and find an 8.5-day discrepancy with the observed period which, they argue, should be attributed primarily to mantle anelasticity. Carton \& Wahr (1986) construct both analytical and numerical models for the pole tide. The results indicate that the departure of the global pole tide from equilibrium is too small to affect the Chandler period and damping noticeably. It is well-known, however, that the pole tide in the North Sea is anomalously large.

In this paper, we use a specific anelastic model to numerically estimate the effects of mantle anelasticity on the theoretical period, $Q_{w}$ and ellipticity of the Chandler wobble based on the perturbation theory. Using the polar motion data determined by modern geodetic techniques, the ellipticity and orientation of the semimajor axis on the wobble path are computed and compared with theoretical values. The results show that the theoretical Chandler period and $Q_{w}$ are in good agreement with most astrometric observations. Mantle anelasticity is likely to be the most important dissipative source of wobble energy. We find that the effect of anelasticity on the ellipticity of the wobble path is negligible.

\section{Effects of anelasticity on the Chandler period and damping}

\subsection{Direct perturbation}

The effects of mantle anelasticity on the Chandler eigenfrequency $\omega_{0}$ can be described by the complex perturbations in the elastic moduli, in which the real parts change the Chandler period and the imaginary parts induce dissipation of the deformation energy. We assume that there is no bulk dissipation and the perturbation $\delta \mu$ in the shear modulus $\mu_{0}$ is only dependent on radius. Using a scalar function $M$ of radius, the first order perturbation $\delta \omega$ to the eigenfrequency of the elastic Earth is given by (Smith \& Dahlen, 1981)

$$
\frac{2 \delta \omega}{\omega_{0}}=\int_{0}^{a}\left(\frac{\delta \mu}{\mu_{0}}\right) \omega_{0} M r^{2} d r
$$

where $a$ is the mean radius of the Earth. Smith \& Dahlen (1981) have proven that the Chandler period $T_{0}$ of an elastic Earth with an equilibrium pole tide is about 426.7 days. Using (1) and $\delta \mu$ obtained by substituting the stratified model PREM (Dziewonsky \& Anderson 1981) into Zschau's rheological model (Zschau $\&$ Wang 1985), We find the effects of anelasticity on the Chandler period and damping. Our results are $\delta T=9.0$ days and $Q_{w}=77$ respectively.

\subsection{Indirect perturbation}

The response of the anelastic Earth to the equilibrium pole tide will also affect the Chandler period and damping due to the perturbations in the associated loading Love numbers of the elastic Earth. We write the variation in centrifugal potential associated with the wobble as

$$
U=\sqrt{\frac{8 \pi}{15}} \Omega^{2} a^{2}\left(m_{1} Y_{21}^{c}+m_{2} Y_{21}^{s}\right)=U_{1} Y_{21}^{c}+U_{2} Y_{21}^{s},
$$


where $\Omega$ is the Earth's mean angular velocity, $m_{1}$ and $m_{2}$ are the equatorial components of the position of the rotation axis, $Y_{21}^{c}$ and $Y_{21}^{s}$ are the real harmonics of degree 2 , order 1 . The equilibrium pole tide has the form

$$
\begin{array}{r}
H=\left\{\frac{\gamma_{2}}{g}\left(U_{1} Y_{21}^{c}+U_{2} Y_{21}^{s}\right)+\sum_{n=0}^{\infty} \frac{3}{2 n+1} \frac{\phi_{m}}{\rho} \gamma_{n}^{\prime}\left[H_{n 0} Y_{n 0}+\right.\right. \\
\left.\left.\sum_{m=1}^{n}\left(H_{n m}^{c} Y_{n m}^{c}+H_{n m}^{s} Y_{n m}^{s}\right)\right]+c\right\} \varepsilon,
\end{array}
$$

where $\rho$ and $\rho_{w}$ are the mean density of the Earth and ocean, $\gamma_{2}=1+k_{2}-h_{2}$ and $\gamma_{n}^{\prime}=1+k_{n}^{\prime}-h_{n}^{\prime}$ are the combinations of the Love numbers and loading Love numbers, respectively, $c$ is an arbitrary constant, and $\varepsilon$ is the ocean function. If cross-coupling of different degree $n$ and order $m$ in the tidal height expansion is ignored, from (3) we have

$$
\left[\begin{array}{l}
H_{21}^{c} \\
H_{21}^{s}
\end{array}\right]=\frac{1}{g} \mathbf{W} \cdot\left[\begin{array}{l}
U_{1} \\
U_{2}
\end{array}\right],
$$

where the coefficient matrix $\mathbf{W}$ is

$$
\mathbf{W}=\gamma_{2}\left[\mathbf{I}-\alpha \gamma_{2}^{\prime} X\right]^{-1} \cdot \mathbf{X}
$$

in which $\alpha=3 \rho_{w} / 5 \rho, \mathrm{I}$ is the unit matrix, $\mathrm{X}$ is the matrix related to the ocean function. Using the ocean function coefficients (Balmino et al. 1973) gives

$$
X_{11}=0.76888, \quad X_{12}=X_{21}=-0.01524, \quad X_{22}=0.60819 .
$$

Let $\mathbf{P}=\alpha\left(1+k_{2}^{\prime}\right) \mathbf{W}$. For an elastic Earth with a fluid core, the effect of the equilibrium pole tide on the polar motion can be calculated from the Liouville equations

$$
\begin{aligned}
& \dot{m}_{1}+\left(\omega_{e}-\frac{\Omega^{2} a^{5}}{3 G A_{m}} P_{22} \Omega\right) m_{2}-\frac{\Omega^{2} a^{5}}{3 G A_{m}} P_{21} \Omega m_{1}=0, \\
& \dot{m}_{2}-\left(\omega_{e}-\frac{\Omega^{2} a^{5}}{3 G A_{m}} P_{11} \Omega\right) m_{1}+\frac{\Omega^{2} a^{5}}{3 G A_{m}} P_{12} \Omega m_{2}=0,
\end{aligned}
$$

where $\omega_{e}=\Omega / 396.9$ is the eigenfrequency of an elastic and oceanless Earth, $G$ is the gravitational constant, and $A_{m}$ is the equatorial moment of inertia of the mantle. The corresponding eigenfrequency of $(7)$ is $\omega_{0}=\omega_{e}+\Delta \omega$, in which the effect of the equilibrium pole tide on the Chandler eigenfrequency of the elastic Earth is approximately

$$
\Delta \omega=-\frac{1}{2} \operatorname{tr} \mathbf{P} \frac{\Omega^{2} A^{5}}{3 G A_{m}} \Omega
$$

where $\operatorname{tr} P$ is the trace of the matrix $P$. The perturbation $\Delta T$ in the Chandler period corresponding to $\Delta \omega$ is 29.8 days (Smith \& Dahlen, 1981). 
Let $\delta \gamma_{2}$ and $\delta \gamma_{2}^{\prime}$ be the perturbations in $\gamma_{2}$ and $\gamma_{2}^{\prime}$ due to anelasticity. Using models PREM and Zschau, we have $\delta \gamma_{2}=-0.0089+\imath 0.0026$ and $\delta \gamma_{2}^{\prime}=$ $-0.0163-\imath 0.0047$. Noting that $k_{2}^{\prime}=k_{2}-h_{2}$, the perturbation in $P$ due to $\delta \gamma_{2}$ and $\delta \gamma_{2}^{\prime}$ is

$$
\delta \mathbf{P}=2 \frac{\delta \gamma_{2}}{\gamma_{2}} \mathbf{P}+\left[\alpha \gamma_{2} L \mathbf{I}+\frac{1}{\gamma_{2}^{2}}(\operatorname{tr} \mathbf{P}) \mathbf{P}\right] \delta \gamma_{2}^{\prime}
$$

where $L=-\alpha \gamma_{2}|X| /|D|$ in which $|X|$ and $|D|$ are the determinants of the matrices $\mathbf{X}$ and $\left(\mathbf{I}-\alpha \gamma_{2} \mathbf{X}\right)$ respectively. Then the perturbation in the eigenfrequency can be approximately written as

$$
\delta(\Delta \omega)=-\frac{1}{2} \operatorname{tr}(\delta \mathbf{P}) \frac{\Omega^{2} a^{5}}{3 G A_{m}} \Omega .
$$

The indirect effect of anelasticity on the Chandler period and $Q_{w}$ are $\delta(\Delta T)=-0.9$ day and $\delta Q_{w}=913$. We have also performed a simple estimate in which the cross-coupling effect of different $n$ and $m$ is included. The result indicates that it is too small to affect our calculation.

Carton \& Wahr (1986) show that the effects of the non-equilibrium portion of the pole tide on the Chandler $T_{w}$ and $Q_{w}$ are $0.04-0.38$ day and 1300-11000 respectively. We can conclude that the effects of the non-equilibrium pole tide on the Chandler wobble are much smaller than those of the anelastic perturbation of the equilibrium pole tide, and mantle anelasticity is likely to be the most important dissipative source of the wobble energy.

The final estimate of the theoretical Chandler period, if we note that the indirect effect is to shorten the period, is $T_{w}=434.8$ sidereal days. The quality factor corresponds to $Q_{w}=71$ because both anelastic effects induce the wobble energy dissipation. Comparing with the observed $T_{w}$ and $Q_{w}$ in Table 1 , we see that the theoretical $T_{w}$ and $Q_{w}$ agree well with most astrometric observations.

Table 1. Comparison between observed and theoretical $T_{w}$ and $Q_{w}$.

\begin{tabular}{l|c|c}
\hline \hline Author & Period(days) & $Q_{w}$ (range) \\
\hline Jeffeys (1968) & $433.2 \pm 3.4$ & $61(37,193)$ \\
Wilson \& Haubrich (1976) & $434.0 \pm 2.5$ & $100(50,400)$ \\
Ooe (1978) & $434.8 \pm 2.0$ & $96(50,300)$ \\
Kuehne et al. $(1996)$ & $439.5 \pm 1.2$ & - \\
Furuya \& Chao (1996) & $433.7 \pm 1.8$ & $49(35,100)$ \\
\hline Theoretical values of this paper & 434.8 & 71 \\
\hline
\end{tabular}

\section{Theoretical ellipticity of the Chandler wobble}

According to the gravity model GEM-T3 (Lerch et al. 1994), the normalized geopotential coefficients of degree 2 are 


$$
\begin{array}{cc}
\bar{C}(20)=-0.48416510 \times 10^{-3}, & \bar{C}(22)=0.24390658 \times 10^{-5} \\
\bar{S}(22)=-0.14000946 \times 10^{-5}, & \bar{C}(21)=\bar{S}(21)=0 .
\end{array}
$$

Let the dynamic ellipticity of the Earth be $H^{\prime}=0.0032739935$. Using these six values gives the six values of the second-degree inertia tensor. We now rotate the reference frame relative to the $Z$ axis, so that the directions of the axes coincide with those of the Earth's principal moments of inertia. Then the principal moments of inertia are

$$
A^{\prime}=0.32959368 M_{e} a^{2}, \quad B^{\prime}=0.32959002 M_{e} a^{2}, \quad C=0.33067447 M_{e} a^{2},
$$

where $M_{e}$ is the mass of the Earth. The Chandler wobble ellipticity for a rigid Earth with the 3-axis dynamic ellipsoid is

$$
e_{0}=1-\sqrt{\frac{B^{\prime}\left(C-B^{\prime}\right)}{A^{\prime}\left(C-A^{\prime}\right)}}=0.0017 .
$$

The world's oceans are asymmetrically distributed upon the Earth's surface so the pole tide makes the Chandler wobble path an ellipse with a small flattening. For an elastic Earth, the theoretical ellipticity $e$ can be easily determined (Munk \& MacDonald 1960):

$$
e=\frac{1}{2} \frac{\Omega}{\omega_{e}}\left(Z_{1}-Z_{2}\right)
$$

The semimajor axis is directed to east longitude $\lambda$. That is

$$
\tan 2 \lambda=\frac{2 R}{Z_{1}-Z_{2}}
$$

where

$$
\begin{gathered}
Z_{1}=\frac{\Omega^{2} a^{5}}{3 G A_{m}} P_{11}=1.8845 \times 10^{-4}, \\
Z_{2}=\frac{\Omega^{2} a^{5}}{3 G A_{m}} P_{22}=1.4395 \times 10^{-4}, \text { and } \\
R=\frac{\Omega^{2} a^{5}}{3 G A_{m}} P_{12}=\frac{\Omega^{2} a^{5}}{3 G A_{m}} P_{21}=-4.2060 \times 10^{-6} .
\end{gathered}
$$

Substituting (16) into equations (14) and (15) yields

$$
e=0.0088, \lambda=-5^{\circ} .4 \text {. }
$$

Comparing (17) with (13), we can find that the effect of the Earth's 3-axis feature on the wobble ellipticity is about 19 per cent of that of the equilibrium pole tide.

The effects of mantle anelasticity on the Chander wobble ellipticity can be modelled by introducing the quality factor $Q_{w}$. Then the complex components $\tilde{m}_{1}$ and $\tilde{m}_{2}$ of the wobble are approximately 


$$
\tilde{m}_{1}=m_{1}+Q_{w}^{-1} m_{2} \quad \tilde{m}_{2}=-Q_{w}^{-1} m_{1}+m_{2}
$$

Putting (18) into (7), the Liouville equations become

$$
\begin{aligned}
& \left.\dot{\tilde{m}}_{1}+\left[\omega_{e}-\left(Z_{2}-R Q_{w}^{-1}\right) \Omega\right] \tilde{m}_{2}-\left[R+\frac{\omega_{e}}{\Omega}-Z_{2}\right) Q_{w}^{-1}\right] \Omega \tilde{m}_{1}=0 \\
& \left.\dot{\tilde{m}}_{2}-\left[\omega_{e}-\left(Z_{1}-R Q_{w}^{-1}\right) \Omega\right] \tilde{m}_{1}+\left[R-\frac{\omega_{e}}{\Omega}-Z_{1}\right) Q_{w}^{-1}\right] \Omega \tilde{m}_{2}=0
\end{aligned}
$$

The theoretical pole path ellipticity $\tilde{e}$ and semimajor axis orientation $\tilde{\lambda}$ of the anelastic Earth can be approximated as

$$
\begin{gathered}
\tilde{e}=\frac{1}{2} \frac{\Omega}{\omega_{e}}\left[\left(Z_{1}-Z_{2}\right)-2 R Q_{w}^{-1}\right], \\
\tan 2 \tilde{\lambda}=\frac{2 R}{Z_{1}-Z_{2}}+Q_{w}^{-1} .
\end{gathered}
$$

Using the theoretical $Q_{w}=71$, we have

$$
\tilde{e}=0.0089, \tilde{\lambda}=-5.0 \text {. }
$$

The effects of anelasticity increase the Chandler wobble ellipticity by about $2.4 \times 10^{-5}$ and change the orientation of semimajor axis by 0.4 in the opposite direction. Both of them are too small to be detected by astrometric observations.

\section{Detection of ellipticity}

To detect the theoretical ellipticity and orientation of semimajor axis, we analyze the SPACE92 polar motion series for the period 1976-1992 (Gross 1993). The parameters of the Chandler wobble, annual and secular polar motion are estimated respectively based on the deconvolution method in Fourier analysis (Gao 1994). Then, the parameters of the Chandler wobble are reduced to the same epoch. After taking the average, the components of the Chandler wobble can be derived

$$
\bar{x}=0.11858 \cos \left(\omega_{w} t-12.52\right) \quad \bar{y}=0.11761 \sin \left(\omega_{w} t-12 \circ 56\right) .
$$

From (22) the observed wobble ellipticity and orientation of the semimajor axis are

$$
\tilde{e}=0.0083, \quad \tilde{\lambda}=-12.5 \text {. }
$$

The observed ellipticity and orientation of semimajor axis agree with the theoretical values. We can deduce that the pole tide in the globe should be close to equilibrium. 


\section{Discussion and conclusions}

We have estimated the effects of mantle anelasticity on the Chandler wobble period, damping, ellipticity and orientation of the semimajor axis based on perturbation principles. We find that the Chandler period of the elastic Earth is lengthened 9.0 days due to anelasticity. The additional response of the anelastic Earth to the equilibrium pole tide makes the Chandler period decrease by 0.9 days and the quality factor $Q_{w}$ decrease by about 8 per cent. The theoretical Chandler period and $Q_{w}$ are 434.8 sidereal days and 71 respectively for an elliptical and rotating Earth with an anelastic mantle, a fluid core and an equilibrium pole tide. These are in good agreement with the observed period and $Q_{w}$. We can conclude that mantle anelasticity is likely to be the most important dissipative source of wobble energy. The effects of anelasticity on the Chandler wobble ellipticity and orientation of the semimajor axis are too small to be detected by astrometric observations. Analysis of the SPACE92 polar motion series shows that the observed ellipticity and orientation of the semimajor axis agree with the theoretical values. We can deduce that the global pole tide should be close to equilibrium.

Acknowledgments. The authors gratefully acknowledge the support of K. C. Wong Education Foundation, Hong Kong. This research is also supported by the National Natural Science Foundation of China under grant 49774229, and by the Climbing Program of China under grant 970231003 .

\section{References}

Balmino, G., K. Lambeck, and W. M. Kaula, 1973, A spherical harmonic analysis of the Earth's topography, J. Geophys. Res., 78, 478-481.

Carton, J.A. and J. M. Wahr, 1986, Modelling the pole tide and its effect on the Earth's rotation, Geophys. J. R. Astron. Soc., 84, 121-137.

Dziewonski, A. and D. L. Anderson, 1981, Preliminary reference Earth model, Phys. Earth Planet. Inter., 25, 297-356.

Furuya, M. and B. F. Chao, 1996, Estimation of period and Q of the Chandler wobble, Geophys. J. Int., 127, 693-702.

Gao, B., 1994, Is the Chandler wobble stable, Science in China (B), 37, 84-93.

Gross, R. S., 1993, A combination of Earth orientation data: SPACE92, in Earth Orientation, Reference Frames and Atmospheric Excitation Functions Submitted for the 1992 IERS Annual Report, IERS Tech. Note 14, edited by P. Charlot, pp. C1-C8, Obs. de Paris, Paris.

Jeffreys, H., 1968, The variation of latitude, Mon. Not. R. Astr. Soc., 141, $255-268$.

Kuehne, J., C. R. Wilson, and S. Johnson, 1996, Estimates of the Chandler wobble frequency and Q, J. Geophys. Res., 101, 13573-13579.

Lerch, F. J. et al., 1994, A geopotential model for the Earth from satellite tracking, altimeter, and surface gravity observations: GEM-T3, J. Geophys. Res., 99, 2815-2839. 
Munk, W. H. and G. J. F. MacDonald, 1960 The Rotation of the Earth, Cambridge University Press.

Ooc, M., 1978, An optimal complex AR.MA model for the Chandler wobble, Geophys. J. R. Astr. Soc., 53, 445-457.

Smith, M. L. and F. A. Dahlen, 1981, The period and Q of the Chandler wobble, Geophys. J. R. Astron. Soc., 64, 223-282.

Wilson, C. R. and R. A. Haubrich, 1976, Meteorological excitation of the Earth's wobble, Geophys. J. R. Astr. Soc., 46, 707-743.

Zschau, J. and R. Wang, 1985, Imperfect elasticity in the Earth's mantle, Implications for the Earth tides and long period deformations, in Proceedings of 10th International Symposium on "Earth's Tides," Madrid, Spain, 1985, pp. 379-382, Cons. Super. De Invest. Cient., Madrid. 